- Large variety of forecasting products required for electricity markets:
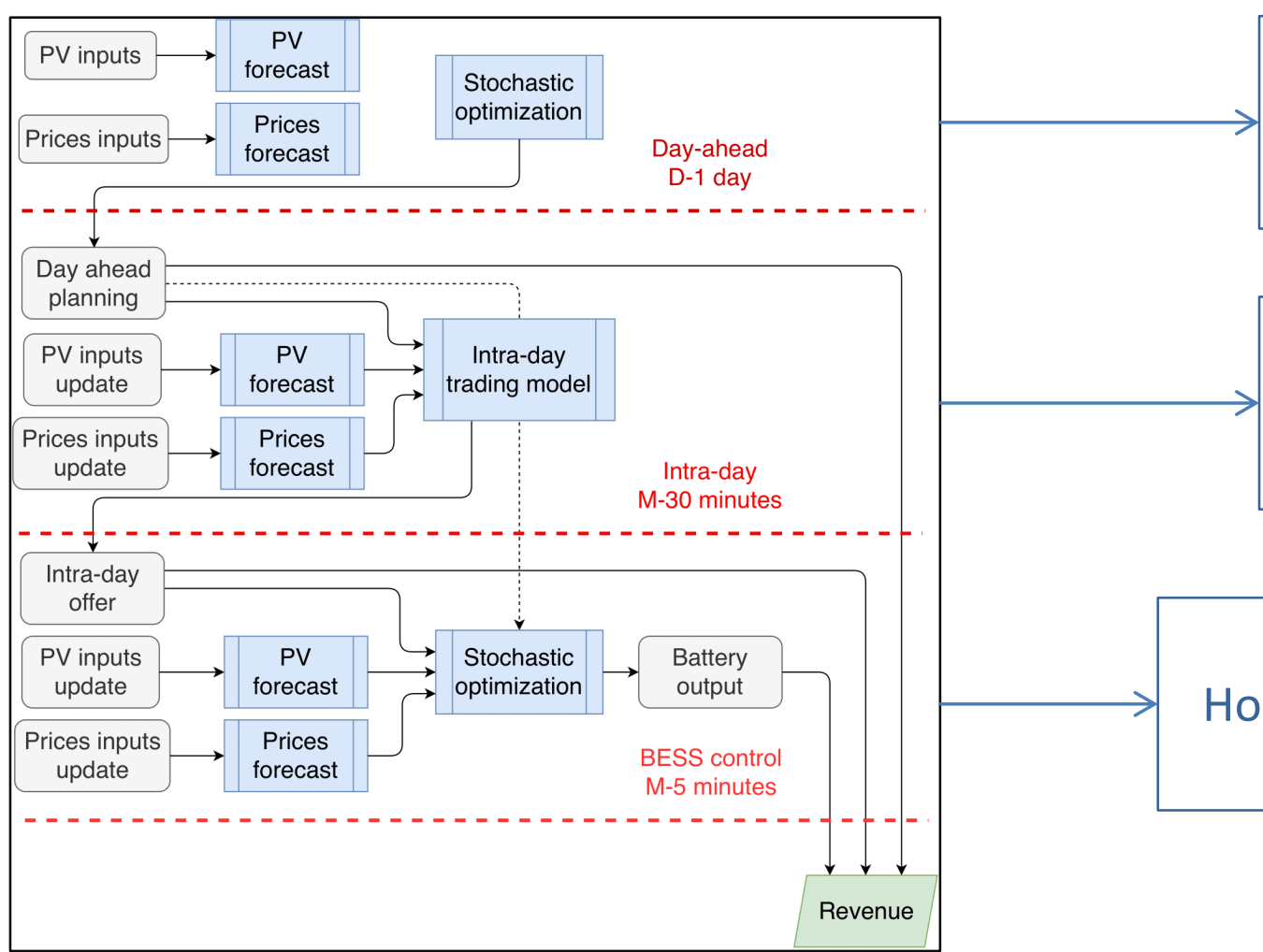

Day-ahead schedule:

Horizon +12 to +36 hours

Resolution $\sim 1$ hour

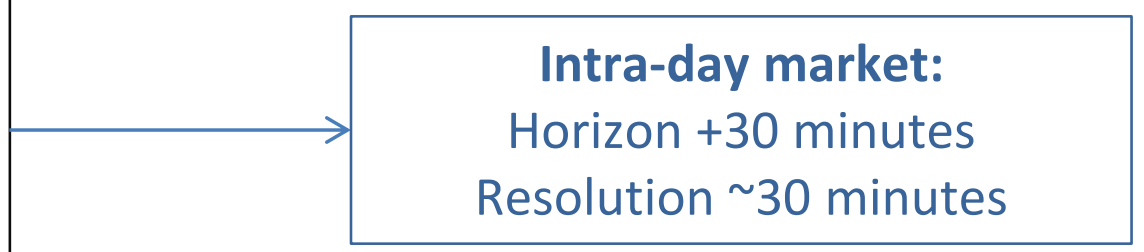

\section{Storage planning:}

Horizon as close to real-time as possible Resolution as high as possible 
- $\quad$ PV power forecasting models are often designed for one specific horizon/resolution

- In this work we propose a single model to cover all PV forecasting products

- This effectively simplifies the usage of PV power forecasts in several decision-making processes 
- Proposition based on the Analog Ensemble (AnEn) method

$>$ Combine heterogenous sources of input

$>$ Forecasts adapted to market time frames:

- Provide both intra-day and day-ahead accurate forecasts

- Provide forecasts with both high (i.e. 5 minutes) and low (i.e. 30 minutes) temporal resolutions

- Provide forecasts starting at any time of the day

$>$ As fast as possible

Probabilistic forecasts for decision under uncertainty

\section{Already present in the state-of-the-art}

To implement 


\section{Methodology}

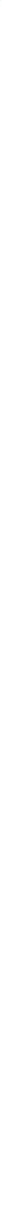




\section{Methodology}

- To achieve short-term forecasts:

statistical learning models

$>$ Measurements for very short-term

$>$ Satellite data for short-term

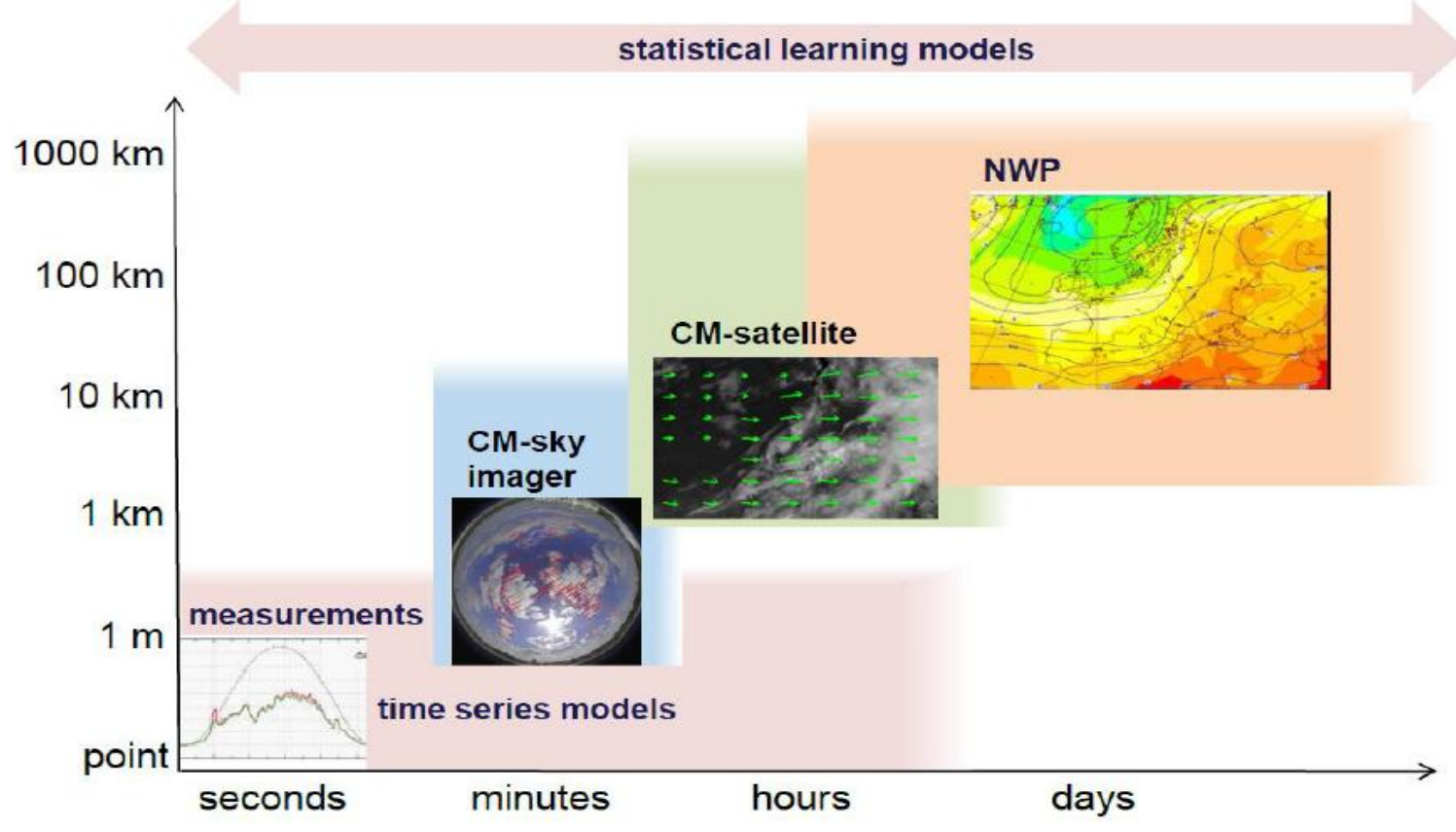




\section{Methodology}

- Standard AnEn model:

- The weights $w_{i}$ are optimized on a training set ("wrapper")

$>$ We propose a "filter" approach to compute the weights directly from the history without an optimization loop 


\section{Methodology}

- When $h$ is the forecast horizon:

n $T$

- The weights $w_{i}^{h}$ are dependent on the forecast horizon. 


\section{Methodology}

- Satellite data: Estimation of GHI time series for each pixel from geostationary satellite images following [Blanc2011]

$>$ Very large number of redundant features

$>$ Selection of representative pixels based on MI with the measurements
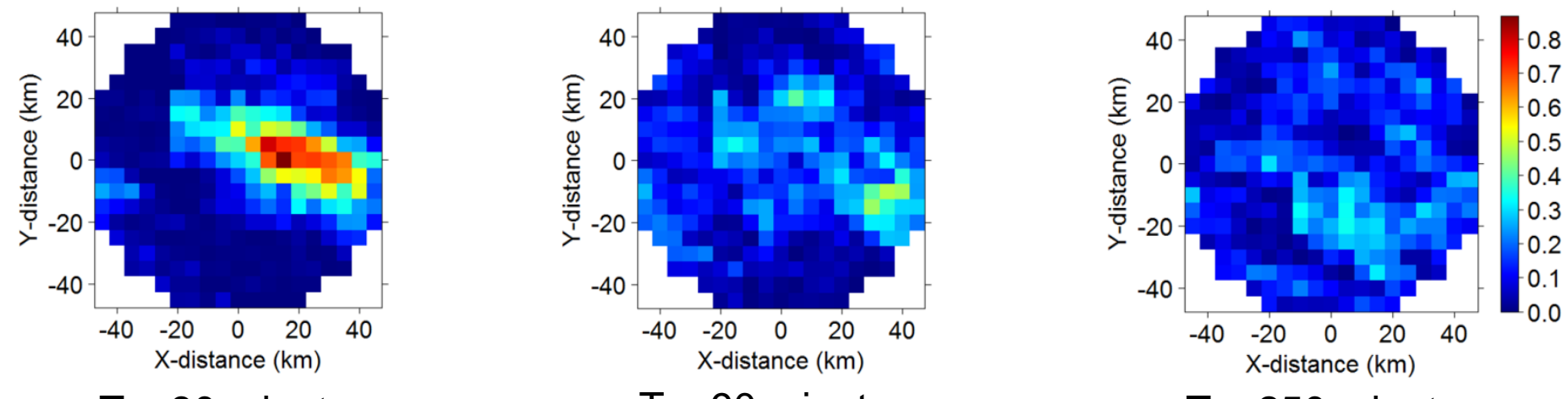

$\mathrm{T}+30$ minutes

$\mathrm{T}+90$ minutes

$\mathrm{T}+250$ minutes

[Blanc2011]: Philippe Blanc et al., The HelioClim project : Surface solar irradiance data for climate applications, In: Remote Sensing 3.2 (2011) 


\section{Methodology}

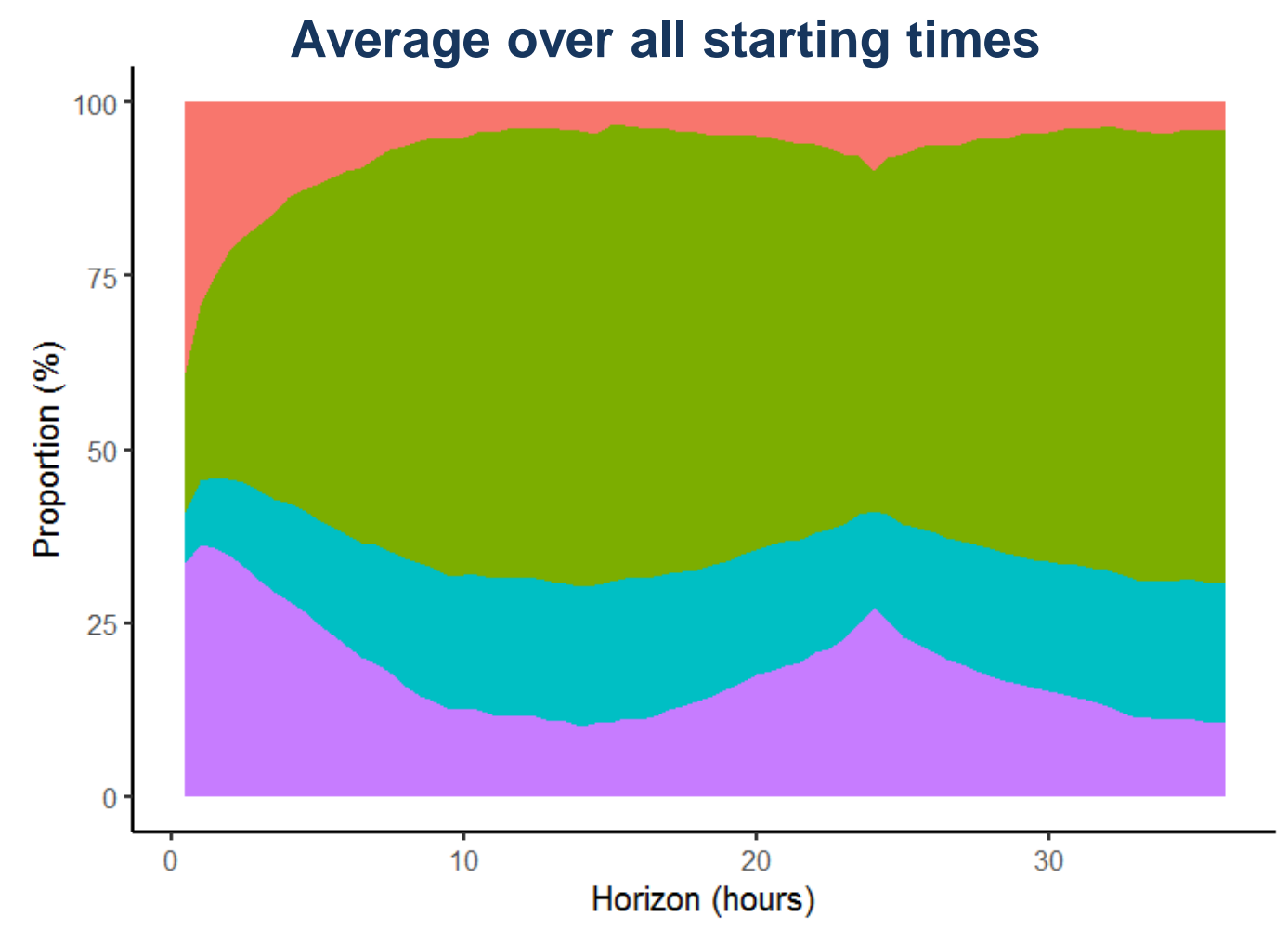

Features

Measures

NWP

CLS

Satellite

\section{AM}

12 AM

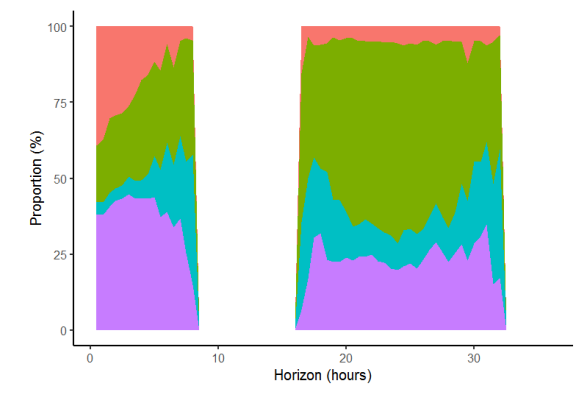

17 PM

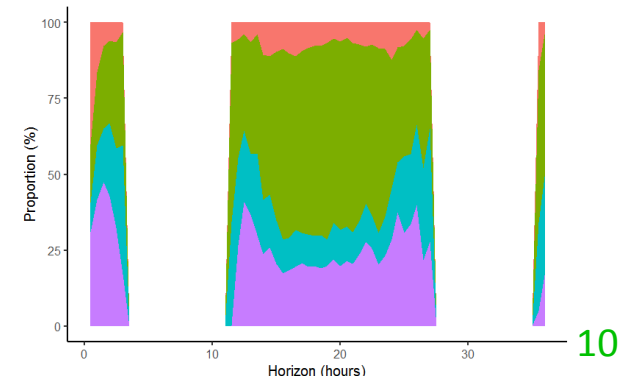


- Evaluation of the AnEn model on a portfolio composed of 12 PV plants ranging from 2 to $10 \mathrm{MW}$.

- Measurements with 5- and 30-minute resolutions are used

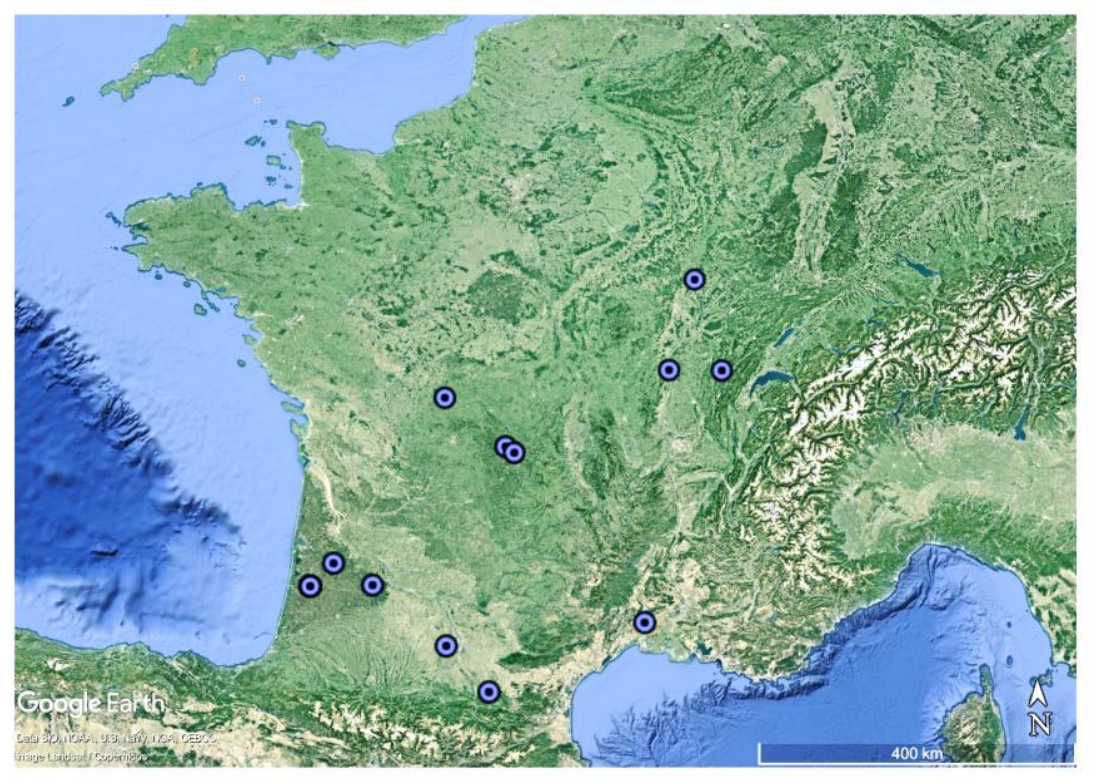

- Benchmark models are implemented:

> Day-ahead forecasts: Quantile Random Forest (QRF) and Bayesian Automatic Relevance Determination (ARD)

$>$ Intra-day forecasts: Clear-sky index persistence and integrated auto-regressive (ARIMA) model 


\section{Result - 30 minute resolution}

- Forecast performance for day-ahead forecasts (30-minute resolution, plant $\left.n^{\circ} 4\right)$ :

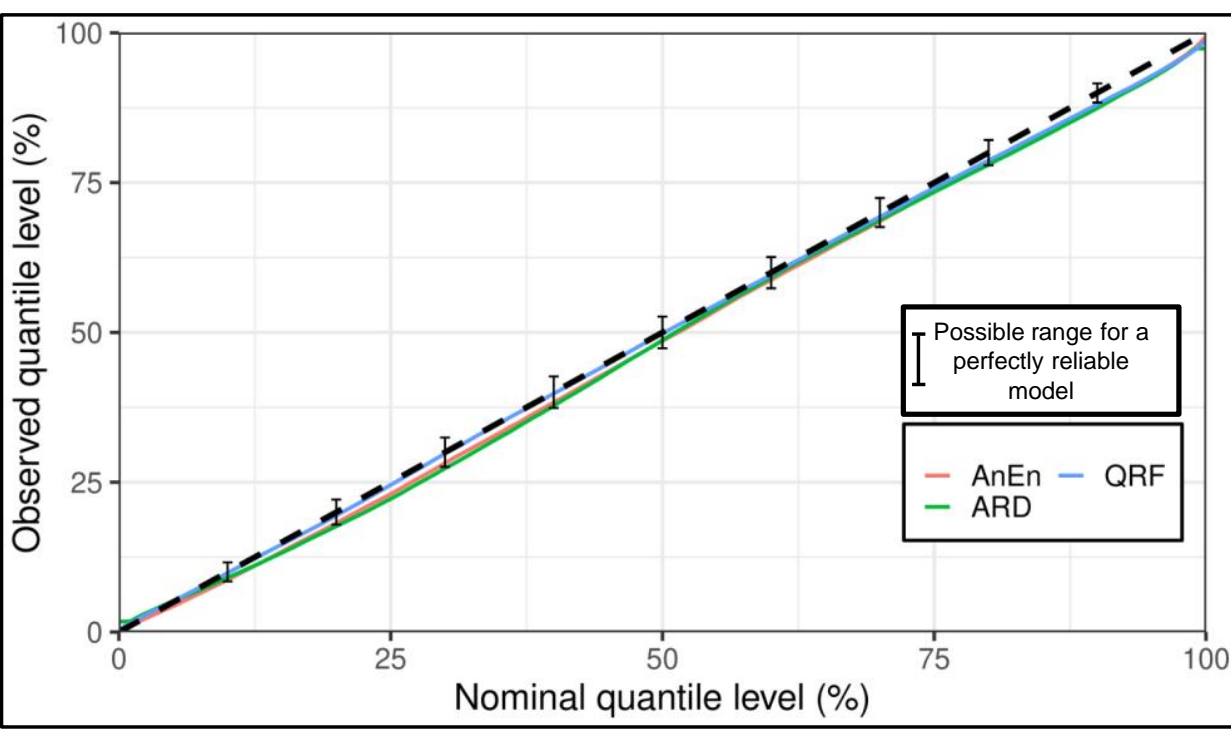

Reliability diagram: nominal vs. observed quantiles

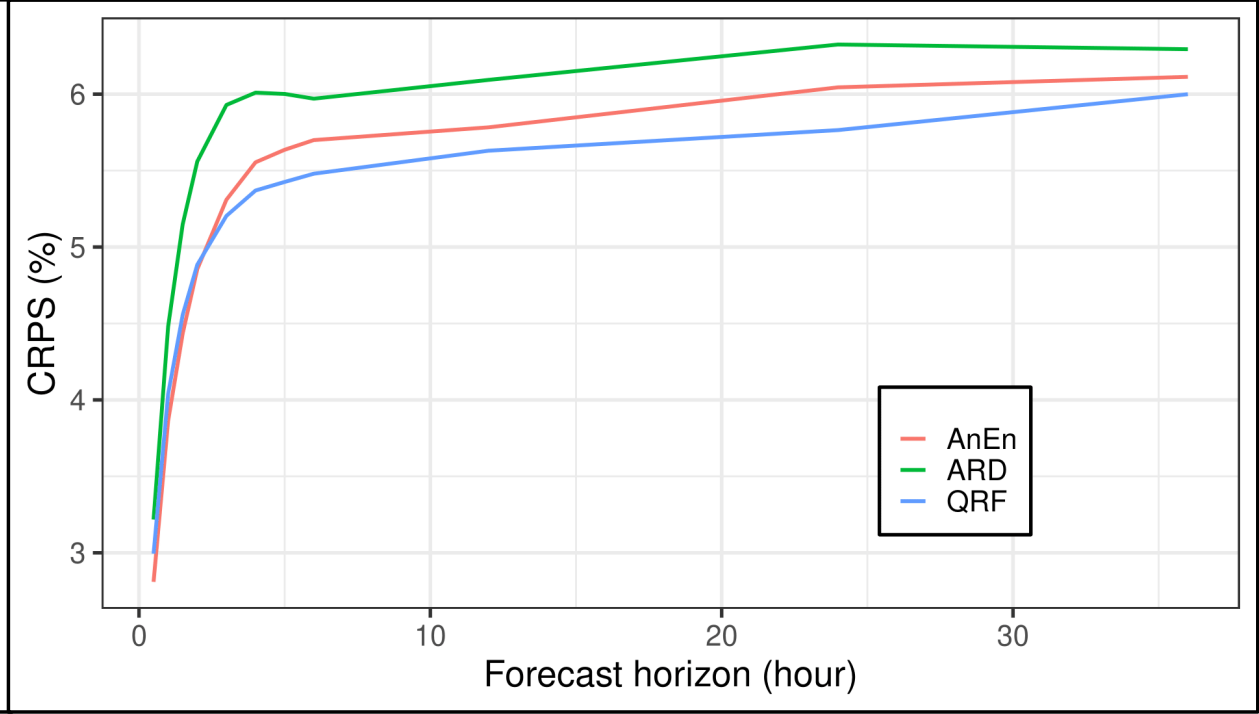

CRPS: Continuous Ranked Probability Score, normalized by installed PV capacity 


\section{Result - 5 minute resolution}

- $\quad$ Forecast performance for intra-day 5-minute forecasts. QRF and ARD models are discarded due to computational cost:

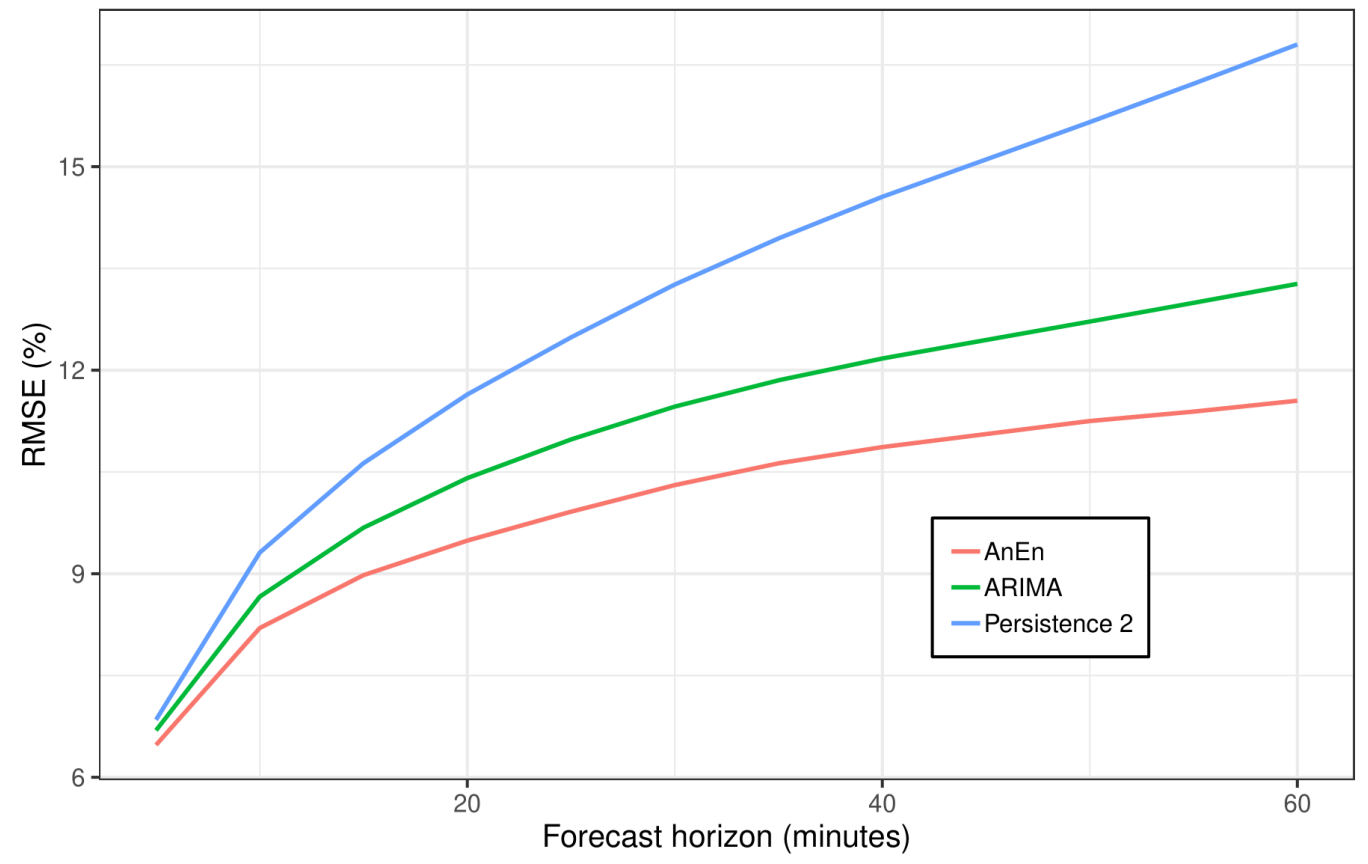

RMSE: Root Mean Square Error, normalized by installed power 
- Computing time required for providing a forecast for a given horizon in seconds

\begin{tabular}{c|cc|cc}
\hline & \multicolumn{2}{|c|}{ 30-minute resolution } & \multicolumn{2}{c}{ 5-minute resolution } \\
& Training & Forecasting & Training & Forecasting \\
\hline AnEn & - & 1.87 & - & 8.77 \\
Persistence 1 & - & $5 \mathrm{e}-3$ & - & $6 \mathrm{e}-3$ \\
Persistence 2 & - & $5 \mathrm{e}-3$ & - & $6 \mathrm{e}-3$ \\
ARIMA & $9.2 \mathrm{e}-2$ & $2.5 \mathrm{e}-7$ & $10 \mathrm{e}-2$ & $3.7 \mathrm{e}-3$ \\
QRF & 4.26 & $1.3 \mathrm{e}-2$ & 68.0 & $4 \mathrm{e}-2$ \\
ARD & 10.75 & $10 \mathrm{e}-3$ & 154 & $1 \mathrm{e}-3$ \\
\hline
\end{tabular}


- A single forecasting model that can quickly provide accurate forecast for temporal resolutions from 5-minute to 1-hour, and horizon from 5 minutes to 3 hours

- Additional types of data could be used:

$>$ All-sky imagers

$>$ Neighboring plants or weather stations

$>$ Infra-red satellite data

$>$ Commercial forecasts from third entities

- More details in: T. Carriere, C. Vernay, S. Pitaval, F.P. Neirac, G. Kariniotakis, A Novel Approach for Probabilistic Photovoltaic Power Forecasting Covering Multiple Time Frames, IEEE Transactions on Smart Grid 